Zijun Hu, Yifeng Ni, Li Huang, Xueyu Jiang, Xiaoyun Yang, Chenglei Li, Qi Wu, Xianyin Zeng and Kuan Liu*

\title{
Meta-linked cationic poly(pyridinylene vinylene) conjugated polyelectrolytes: solution photophysics and fluorescent sensing of metal ions
}

https://doi.org/10.1515/epoly-2018-0022

Received February 1, 2018; accepted April 13, 2018; previously published online May 25, 2018

\begin{abstract}
Two novel poly(pyridinylene vinylene) (PPV)type conjugated polyelectrolytes 2,6-PPYPV-(2+) and 3,5PPYPV-(2+) were synthesized via Heck coupling reaction and characterized by ${ }^{1} \mathrm{H}$ nuclear magnetic resonance (NMR), ultraviolet-visible (UV-Vis) and photoluminescence (PL) spectroscopy. The two polymers consisting of meta-position substituted pyridinylenes as the metal recognition unit and a water-soluble divinyl-benzene derivative in the backbones. 2,6-PPYPV-(2+) and 3,5-PPYPV-(2+) both exhibit strongest fluorescence in methanol and weakest fluorescence in water among common polar solvents. In respect of ion detection, 3,5-PPYPV-(2+) has an excellent identifiability for $\mathrm{Pd}^{2+}$ in methanol with the K_SV value of $1.1 \times 10^{5} \mathrm{M}^{-1}$ while 2,6-PPYPV-(2+) has a good selectivity for $\mathrm{Cu}^{2+}$ and $\mathrm{Hg}^{2+}$ in aqueous solution. And all the Stern-Volmer plots of 2,6-PPYPV-(2+) and 3,5-PPYPV$(2+)$ in fluorescence quenching for metal ions have favorable tendencies. All the results suggest that 2,6-PPYPV-(2+) and 3,5-PPYPV-(2+) are promising materials in the applications of high performance chemosensors for some specific metal ions.
\end{abstract}

Keywords: conjugated polyelectrolytes; fluorescence quenching; pyridine; sensing of metal ions; Stern-Volmer plot.

\section{Introduction}

Conjugated polyelectrolytes (CPEs) have been the hot spot in the area of polymer material science and polymer chemistry over the years. The chemical structure of the

\footnotetext{
*Corresponding author: Kuan Liu, College of Science, Sichuan Agricultural University, Yaan 625014, China,

e-mail: cosmicer@live.cn

Zijun Hu, Yifeng Ni, Li Huang, Xueyu Jiang and Xiaoyun Yang:

College of Science, Sichuan Agricultural University, Yaan 625014,

China

Chenglei Li, Qi Wu and Xianyin Zeng: College of Life Science,

Sichuan Agricultural University, Yaan 625014, China
}

CPEs provides a unique set of properties, including excellent water solubility and convenient process ability, main-chain-controlled exciton and charge transport, variable band gap, tunable light absorption and fluorescence, ionic interactions and aggregation phenomena (1). With the above-mentioned characteristics, these materials have been widely investigated for a variety of optoelectronic applications, such as polymer light-emitting diodes (PLEDs) (2), photovoltaic devices (3) and fluorescent chemosensors (4). In recent years, CPEs have showed great potential as novel chemosensors or biosensors materials due to their fluorescence quenching amplification (5), high sensitivities to analytes (6) and environment-friendly biocompatibility (7). Compared to small molecule fluorescence chemosensors, conjugated polymers as delocalized $\pi$-electronic conjugated "molecular wires" can greatly amplify the fluorescence signal by the facile energy migration along the polymer backbone upon light excitation and the amplification of fluorescent signals resulted from the excitation energy transfer to the chromophore along the whole backbone (8). Therefore, CPEs containing featured recognition sites in their main-chain or side-chain were used for the detection of chemical or bioactive species, such as metal ions (9), DNA/RNA (10), proteins (10), methyl viologen (11), melamine (12), saccharides (13), etc.

In respect of ion detection with polymer chemosensor, pyridine is an excellent ligand to bind transition metal ions with the lone pair electrons of nitrogen filled in the unoccupied orbitals of ions. This process finally leads to a coordination compound being formed and it could change the electronic structures of the polymer main chain which can lead to observable variation or remarkable quenching of fluorescence. Thus, with the pyridine ring as the recognition unit among the backbone, the pyridine-based conjugated polymers have realized sensitive detections of multiple ions (14). However, the common conjugated polymers for the detection of metal ions are basically oil-soluble because the alkoxy-substituted aromatic ring systems are frequently used as the copolymerization unit (15). Thus, the fluorescence sensing properties should be investigated in a weak polar solvent such as tetrahydrofuran. Even so, few publications concerned about CPEs for the detection of ions have been reported (16). In these pioneering works on the 
CPE-based fluorescence chemosensor, the poly(phenylene ethynylene) (PPE) backbone is the most commonly used structure. Although the $\mathrm{C}-\mathrm{C}$ triple bond along these polyelectrolytes can give a satisfactory fluorescence property, the rigid main chain can result in the considerable $\pi$ - $\pi$ aggregation of the polyelectrolytes in polar solvents (17).

As for the detection of metal ions, few cationic CPEs have been reported because of the limitations of synthetic methods (18), and almost all of these cationic polymers were synthesized via the "indirect method". This kind of approach requires a quaternization reaction between the tertiary amine side group and the haloalkane after the palladium-catalyzed coupling copolymerization. Completely positively-charged cationic polyelectrolytes cannot be obtained through this indirect method because not all the amino groups linked to the side-chain of the polymers can be charged in this procedure which can result in a poor solubility. In general, the quaternization degree of the polyelectrolyte which can be obtained from nuclear magnetic resonance (NMR) analysis will remain below $60 \%$ (19). In our previous work, a "direct strategy" was designed and achieved to afford the completely charged cationic polymer (20). In this multi-step synthetic route, a cationic copolymerization monomer $1,4-\mathrm{bis}(3-(\mathrm{N}, \mathrm{N}, \mathrm{N}-$ triethylammonium)-1-oxapropyl)-5-divinyl-benzene dibromide was prepared first via the quaternization reaction followed by Heck-coupling polymerization with another monomer to give novel cationic polymers. This direct strategy can ensure that all the tertiary amine groups can be transformed to quaternary ammonium salt.

Herein, Heck-coupling reaction between metaposition substituted pyridine dihalide derivatives and the reported cationic monomer was implemented to afford two novel cationic pyridine-contained CPEs 3,5-PPYPV(2+) and 2,6-PPYPV-(2+), respectively. The two novel poly(pyridinylene viylene) (PPV)-type CPEs possess better solubility than PPE-type polyelectrolytes. Like with other CPEs, both polymers show remarkable photophysicssolvent dependence. In aqueous solution, the polymers exhibit weak fluorescence due to the $J$-aggregation. On the contrary, the cationic PPYPVs have feeble aggregations in methanol and the polymer solutions emit stronger fluorescence than that of aqueous solution. Furthermore, 2,6-PPYPV-(2+) and 3,5-PPYPV-(2+) both exhibit highly identifiability for $\mathrm{Pd}^{2+}$ in methanol which is similar to the reported works of oil-soluble pyridine-contained PPE conjugated polymer (21). In addition, 2,6-PPYPV-(2+) can discern $\mathrm{Cu}^{2+}$ in methanol and $\mathrm{Hg}^{2+}, \mathrm{Cu}^{2+}$ in aqueous solution as well. In comparison to the ability of 2,6-PPYPV-(2+) for detecting metal ions, 3,5-PPYPV-(2+) shows an excellent recognition capacity for $\mathrm{Pd}^{2+}$ in methanol solution.

\section{Experimental}

\subsection{Measurements}

${ }^{1} \mathrm{H}$ NMR measurements were carried out on a Bruker Avance II- $400 \mathrm{MHz}$ spectrometer (Bruker, Karlsruhe, Germany) with tetramethylsilane as the internal reference. UV-Vis spectra were performed on an AnalytikJena Specord200Plus UV-Vis spectrophotometer (AnalytikJena, Jena, Germany). Photoluminescence (PL) spectra were recorded on a Hitachi F-4600 luminescence spectrophotometer (Hitachi, Tokyo, Japan). All spectra were measured at room temperature.

\subsection{Materials}

All of chemicals were purchased from Aladdin Industrial Corporation (Aladdin, Shanghai, China) and were used directly without further purification.

\subsection{Synthesis of polymers}

Monomer 1 was synthesized from hydroquinone as the primal reactant via five steps. The synthetic details refer to the published literature (20).

\subsubsection{Synthesis of 3,5-PPYPV-(2+)}

Under argon atmosphere, monomer $1(0.578 \mathrm{~g}, 1.00 \mathrm{mmol})$, 3,5-dibromopyridine $(0.237 \mathrm{~g}, 1.00 \mathrm{mmol})$, palladium(II) acetate $(0.014 \mathrm{~g}, 0.06 \mathrm{mmol})$ and tri(o-tolyl)phosphine $(0.073 \mathrm{~g}, 0.24 \mathrm{mmol})$ were dissolved in the mixed solvent (toluene/triethylamine/DMSO $=2 \mathrm{ml} / 4 \mathrm{ml} / 4 \mathrm{ml}$ ). The reaction mixture was stirred at $130^{\circ} \mathrm{C}$ for $6 \mathrm{~h}$ and subsequently cooled to room temperature. The resulting solution was filtered and the filtrate was poured into the mixed solvent (ethyl ether/ethanol). The precipitate was isolated by filtration and then redissolved in deionized water $(10 \mathrm{ml})$. The resulting solution was dialyzed against deionized water for 5 days using a $4 \mathrm{k}$ molecular weight cut-off (MWCO) cellulose membrane. Finally the solvent was removed by rotary evaporation and the crude product was further dried under vacuum with $\mathrm{P}_{2} \mathrm{O}_{5}$ at $30^{\circ} \mathrm{C}$ for 2 days to give a dark brown powder. Yield: $0.2328 \mathrm{~g}, 35.6 \%$. ${ }^{1} \mathrm{H}$ NMR (400 MHz, DMSO-d,,$\left.\delta\right): 9.42-6.91$ (m, 9H), 4.50 (dd, $J=104.2,60.6 \mathrm{~Hz}, 4 \mathrm{H}), 3.88$ (t, $J=60.1 \mathrm{~Hz}, 4 \mathrm{H}), 3.43$ (s, 12H), 1.11 (s, 18H). 


\subsubsection{Synthesis of 2,6-PPYPV-(2+)}

The route is the same to 3,5-PPYPV-(2+) and the product is a dark-brown metallic luster powder. Yield: $0.2860 \mathrm{~g}$, 43.8\%. ${ }^{1} \mathrm{H}$ NMR (400 MHz, DMSO- $\left.d_{6}, \delta\right): 8.90-7.31$ (m, 9H), 7.08-6.98 (m, 1H), $6.03(\mathrm{~d}, J=17.4 \mathrm{~Hz}, 1 \mathrm{H}), 5.42(\mathrm{~d}$, $J=11.1 \mathrm{~Hz}, 1 \mathrm{H}), 4.63(\mathrm{~d}, J=75.5 \mathrm{~Hz}, 4 \mathrm{H}), 3.88(\mathrm{dd}, J=53.6$, $32.6 \mathrm{~Hz}, 4 \mathrm{H}), 3.50$ (d, $J=26.8 \mathrm{~Hz}, 12 \mathrm{H}), 1.52-0.98$ (m, 18H).

As some references have reported, the molecular weight data is not available from common techniques such as gel permeation chromatography (GPC) or light scattering method due to the aggregation phenomenon of CPEs (22). It is difficult to find an appropriate solvent which leads to weak aggregation of CPEs with high concentration to finish the GPC measurement. Furthermore, CPEs may be easily adsorbed on the chromatographic column to give imprecise results. In this investigation, solutions of 2,6-PPYPV-(2+) and 3,5-PPYPV-(2+) were dialyzed by $4 \mathrm{kDa}$ MWCO cellulose membrane. This approach can ensure that the molecular weight of 2,6-PPYPV-(2+) and 3,5-PPYPV-(2+) are more than 4000 (at least eight repeating units).

\subsection{Photophysical properties of polymers}

In order to explore photophysical properties, we measured ultra violet-visible (UV-Vis) spectra and photoluminescence (PL) spectra of these two CPEs in different solvents at the same concentration $\left(2.0 \times 10^{-5} \mathrm{M}\right.$, calculated by repeating units). All the absorption and emission maxima of the polymer solutions are listed in Table 1, respectively.

As shown in Table 1, in the same solvent, 2,6-PPYPV(2+) possesses smaller absorbance maxima compared with 3,5-PPYPV-(2+). In DMSO and N,N-dimethylformamide (DMF), the absorbance maxima of 2,6-PPYPV-(2+) solutions are both $393 \mathrm{~nm}$ while the maxima of 3,5-PPYPV-(2+) solutions are both $400 \mathrm{~nm}$ in these two solvents. The difference of absorbance maxima between the two polymers

Table 1: Optical properties of 2,6-PPYPV-(2+) and 3,5-PPYPV-(2+) in different solvents.

\begin{tabular}{llrrr}
\hline Polymer & Solvent & UV-Vis (nm) & PL (nm) & Stokes shift (nm) \\
\hline 2,6-PPYPV-(2+) & Water & 367 & 462 & 95 \\
& Methanol & 379 & 446 & 67 \\
& DMSO & 393 & 457 & 64 \\
& DMF & 393 & 453 & 60 \\
3,5-PPYPV-(2+) & Water & 381 & 459 & 78 \\
& Methanol & 393 & 447 & 54 \\
& DMSO & 400 & 455 & 55 \\
& DMF & 400 & 449 & 49 \\
\hline
\end{tabular}

can be attributed to the different main-chain structure of the polymers. In addition, these polymers exhibit an obvious blue shift of the maximum absorption in protic solvent (methanol, water) compared to aprotic solvent. This unusual behavior could be attributed to the hydrogen bond which is formed between solvent molecules, pyridine rings and the mutual electrostatic repulsion of positive charges in quaternary ammonium salt groups linked to the benzene rings which leads to an increased torsional angle along the conjugated backbone and a decreased effective conjugation length. This phenomenon is similar to other reported positive charged CPEs $(23,24)$.

In the fluorescence spectra which are shown in Figure 1, both polymers exhibit remarkable fluorescence intensities in DMF, DMSO and methanol. However, with the same concentration, aqueous solutions of the polymers exhibit very weak fluorescence and a red shift of the emission maxima in the spectra compared with other polymer solutions. For this consequence, many published results has confirmed that conjugated polyelectrolyte molecules are aggregated strongly by $\pi-\pi$ stacking which can consume the energy of excited state to emit weak fluorescence with longer wavelength in aqueous solutions $(25,26)$. Thus, these results of spectra prove that 2,6-PPYPV-(2+) and 3,5-PPYPV-(2+) exhibit typical solution photophysical behavior as the CPEs. In addition, as the chemical structure shown in Scheme 1, the backbone of 2,6-PPYPV-(2+) is connected through the $\alpha$-carbon atoms of pyridine ring which become electron deficient due to the electron-withdrawing inductive effects of the adjoining nitrogen atom. But for 3,5-PPYPV-(2+), it is connected through the $\beta$-carbon atoms of pyridine ring which have the highest electron cloud density. Therefore, a stronger charge-transfer effect exists between the pyridine unit and divinyl benzene in 2,6-PPYPV-(2+) rather than 3,5-PPYPV(2+) which leads to a larger Stokes shift of 2,6-PPYPV-(2+). According to all the results of spectra, we confirm that the two polymers, 2,6-PPYPV-(2+) and 3,5-PPYPV-(2+), both have the distinct photophysical-solvent dependence.

\subsection{Sensing properties with metal ions}

\subsubsection{Sensing properties of 2,6-PPYPV-(2+)}

To examine the interaction with metal ions, the responsive properties of 2,6-PPYPV-(2+) were studied by fluorescence spectroscopy in water and methanol at a concentration of $2.0 \times 10^{-5} \mathrm{M}$. In aqueous solution and methanol solution, the fluorescence spectroscopy of 2,6-PPYPV-(2+) with metal ions such as $\mathrm{Co}^{2+}, \mathrm{Cr}^{3+}, \mathrm{Cu}^{2+}, \mathrm{Fe}^{2+}, \mathrm{Fe}^{3+}, \mathrm{Mg}^{2+}, \mathrm{Mn}^{2+}$, 

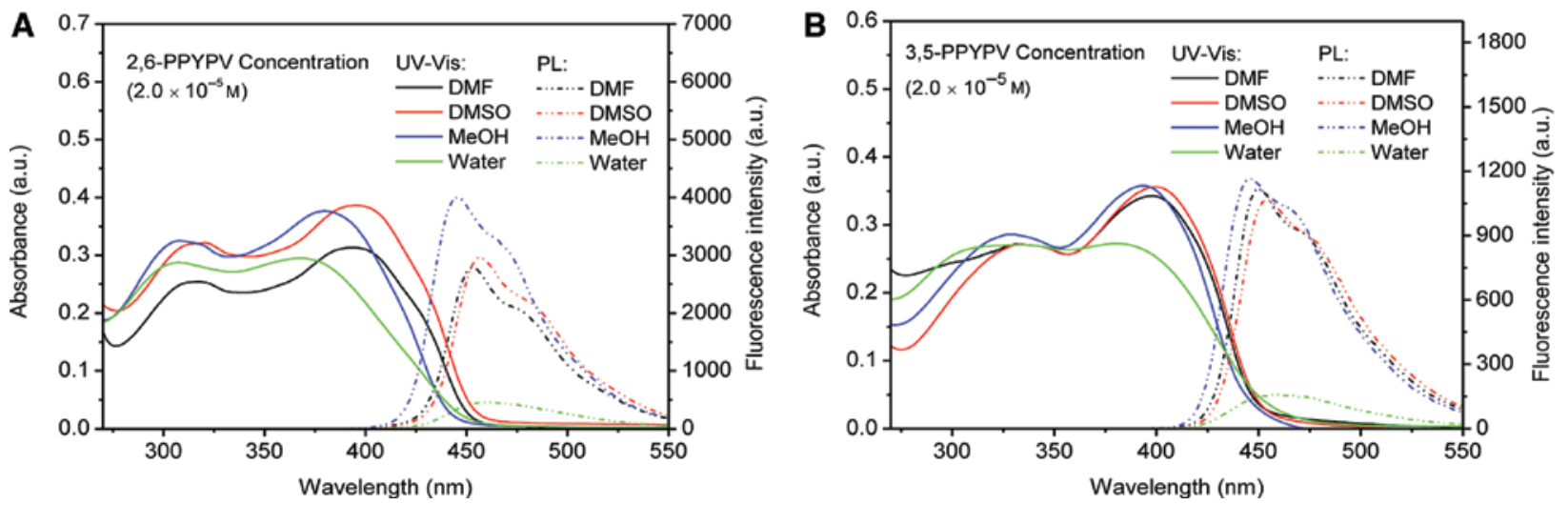

Figure 1: Comparisons of UV-Vis absorption and fluorescence emission for the polymers show solvent effects.

(A) UV-Vis spectra (solid) and PL spectra (dashed) of 2,6-PPYPV-(2+) in different solvents. (B) UV-Vis spectra (solid) and PL spectra (dashed) of 3,5-PPYPV-(2+) in different solvents.<smiles>C=Cc1cc(OCC[N+](CC)(CC)CC)c(C=C)cc1OCC[N+]([B]C)(CC)CC</smiles>

Monomer 1<smiles>Brc1cccc(Br)n1</smiles>

Monomer 2<smiles>Brc1cncc(Br)c1</smiles>

Monomer 3
Monomer $1+$ Monomer 2

$\mathrm{Pd}(\mathrm{OAc})_{2}, \mathrm{P}(\mathrm{o} \text {-tolyl })_{3}$

DMSO, TEA, toluene

$\mathrm{Pd}(\mathrm{OAc})_{2}, \mathrm{P}(\mathrm{o} \text {-tolyl })_{3}$

Monomer $1+$ Monomer 3<smiles>CC[N+](CC)(CC)CCOc1cc(/C=C/c2cccc(C)n2)c(/C=C/C(C)(C)C)cc1OCC[N+](CC)(CC)CC</smiles>

2,6-PPYPV-(2+)<smiles>CC[N+](CC)(CC)CCOc1cc(/C=C/c2cncc(C(C)(C)C)c2)c(/C=C/C(C)(C)C)cc1OCC[N+](CC)(CC)CC</smiles>

3,5-PPYPV-(2+)

Scheme 1: Structures of monomers and synthetic routes of 2,6-PPYPV-(2+) and 3,5-PPYPV-(2+) 
$\mathrm{Ni}^{2+}, \mathrm{Pb}^{2+}, \mathrm{Hg}^{2+}, \mathrm{Sn}^{2+}$ and $\mathrm{Zn}^{2+}$ was investigated. The result of primary responsive properties is shown in Figure 2. (The metal ions solution are at the concentrations which cannot cause any changes of fluorescence intensities, and all the metal ions in ion screening experiments have the same concentration for the same solvent. It is $1.2 \times 10^{-5} \mathrm{M}$ in aqueous solution and $8.0 \times 10^{-6} \mathrm{M}$ in methanol solution for all the metal ions.). In aqueous solution, it can be found that, $\mathrm{Co}^{2+}, \mathrm{Fe}^{2+}, \mathrm{Mg}^{2+}, \mathrm{Mn}^{2+}, \mathrm{Ni}^{2+}, \mathrm{Pb}^{2+}$ and $\mathrm{Zn}^{2+}$ cannot cause fluorescence quenching of the polymer basically. Upon the addition of $\mathrm{Cr}^{3+}, \mathrm{Sn}^{2+}$ and $\mathrm{Fe}^{3+}$, a medium decrease of the fluorescence has occurred. Moreover, $\mathrm{Cu}^{2+}$ exhibits a strong quenching ability in the spectrum and $\mathrm{Hg}^{2+}$ can observably quench the fluorescent emission of 2,6-PPYPV(2+). As shown in Figure 2B, differing from aqueous solution, the sensitivity of 2,6-PPYPV-(2+) for $\mathrm{Cu}^{2+}$ in methanol has been noticeably increased. Moreover, $\mathrm{Pd}^{2+}$ could lead to an amazing fluorescence quenching of 2,6-PPYPV-(2+) in methanol. And $\mathrm{Co}^{2+}, \mathrm{Fe}^{3+}, \mathrm{Cd}^{2+}, \mathrm{Hg}^{2+}, \mathrm{Sn}^{2+}, \mathrm{Zn}^{2+}$ also had strong fluorescence quenching abilities which should be regarded as interference factors, $\mathrm{Ca}^{2+}, \mathrm{Mg}^{2+}, \mathrm{Mn}^{2+}$ still cannot be recognized by 2,6-PPYPV-(2+).

The fluorescence spectra of 2,6-PPYPV-(2+) in water with different concentrations of $\mathrm{Hg}^{2+}$ and $\mathrm{Cu}^{2+}$ are shown in Figure $3 \mathrm{~A}$ and $\mathrm{B}$, respectively. Upon addition of $\mathrm{Hg}^{2+}$, the fluorescence intensity of 2,6-PPYPV-(2+) reduced observably. Furthermore, the interaction of 2,6-PPYPV-(2+) with $\mathrm{Hg}^{2+}$ reached saturation upon addition of 0.6 equiv. $\mathrm{Hg}^{2+}$, because the fluorescence intensity of 2,6-PPYPV-(2+) is nearly invariable even if the concentration of $\mathrm{Hg}^{2+}$ increase continuously. Finally, the fluorescence intensity is reduced from 7488 to 1734 and about $77 \%$ of fluorescence is quenched.

For $\mathrm{Cu}^{2+}$, 2,6-PPYPV-(2+) shows a higher concentration limitation of fluorescence quenching (about $2.6 \times 10^{-5} \mathrm{M}$ ) than that of $\mathrm{Hg}^{2+}$. However, under the saturated concentration of $\mathrm{Cu}^{2+}$, the fluorescence emission is reduced to 3738 and only $52 \%$ of fluorescence is quenched, which is less than that of $\mathrm{Hg}^{2+}$.

Furthermore, we also calculate the fluorescence quenching efficiency according to the Stern-Volmer equation (27):

$$
\mathrm{I}_{0} / \mathrm{I}=\mathrm{K} \_\mathrm{SV}[\mathrm{Q}]+1 \text {, }
$$

where I is the fluorescence intensity, $[\mathrm{Q}]$ is the concentration of quencher, $\mathrm{I}_{0}$ is the intensity at $[\mathrm{Q}]=0 \mathrm{M}$ and $\mathrm{K} \_\mathrm{SV}$ is the Stern-Volmer constant.

At low concentrations of $\mathrm{Hg}^{2+}\left(0-1.0 \times 10^{-6} \mathrm{M}\right)$, a standard linear Stern-Volmer plot is obtained with the $\mathrm{K} \_$SV value about $8.6 \times 10^{5} \mathrm{M}^{-1}$. And at the range of concentration from $1.0 \times 10^{-6} \mathrm{M}$ to $5.0 \times 10^{-6} \mathrm{M}$, another linear Stern-Volmer plot can be fitted as the equation, $\mathrm{y}=0.346270 \mathrm{x}+1.59815$, with a $\mathrm{K}^{\prime} \mathrm{SV}$ value about $3.5 \times 10^{5}$ $\mathrm{M}^{-1}$ (see Figure 3C). The values of $\mathrm{K} \_S V$ and $\mathrm{K}^{\prime}$ SSV are larger than $2.97 \times 10^{4} \mathrm{M}^{-1}$, which is the result for conjugated polyelectrolyte based on benzoselenadiazole in the sensing investigation for $\mathrm{Hg}^{2+}$ (28).

On the other hand, at the full range of concentration with $\mathrm{Cu}^{2+}\left(0-2.6 \times 10^{-5} \mathrm{M}\right)$, the Stern-Volmer plot also has a linear interrelationship as shown in Figure 3D. At the low concentrations $\left(0-5.0 \times 10^{-6} \mathrm{M}\right)$, a standard linear SternVolmer plot is exhibited with the $\mathrm{K} \_$SV value of $1.1 \times 10^{5} \mathrm{M}^{-1}$ and it is certain that there is a turn of slope at the concentration of $5.0 \times 10^{-6} \mathrm{M}$ (see Figure 3D). Thus, another SternVolmer plot is fitted as the equation, $\mathrm{y}=0.029 \mathrm{x}+1.36965$. Otherwise, the plot of fluorescence quenching presents a slightly downward curvature, suggesting the presence of the fractional accessibility of polymer molecules during the interaction with metal ions (29). According to
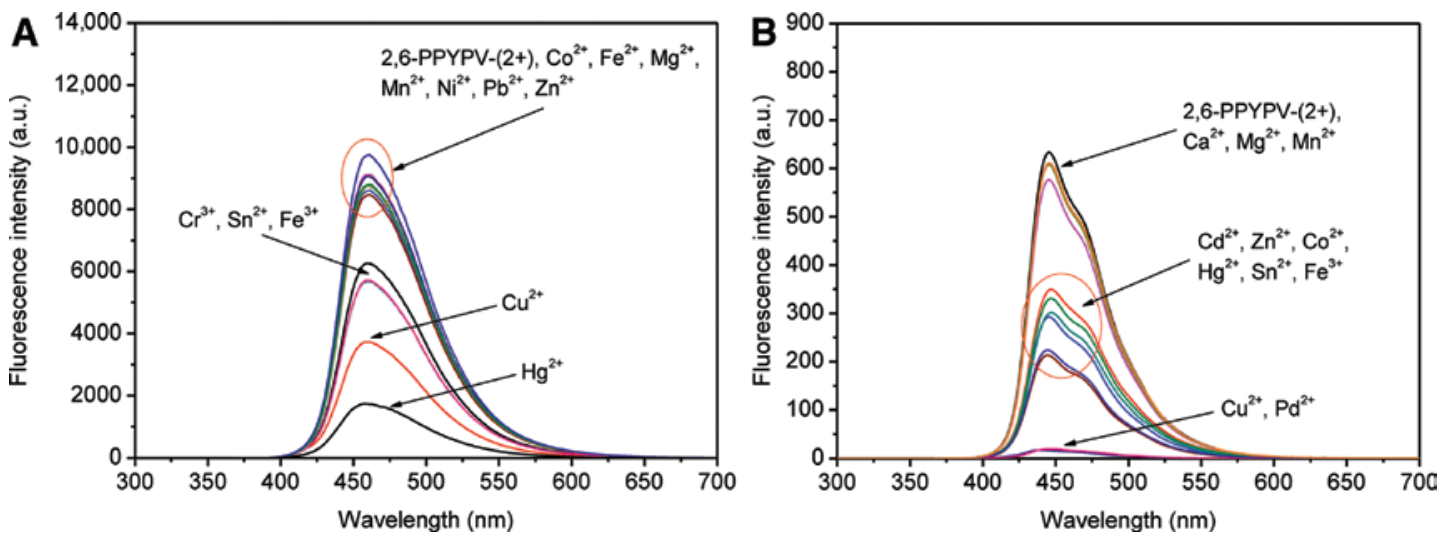

Figure 2: The fluorescence intensities of 2,6-PPYPV-(2+) are drastically decreased by $\mathrm{Cu}^{2+}$ and $\mathrm{Hg}^{2+}$ in aqueous solution and $\mathrm{Cu}^{2+}$ and $\mathrm{Pd}^{2+}$ in methanol solution.

(A) Fluorescence spectra of 2,6-PPYPV-(2+) in aqueous solution with different metal ions. (B) Fluorescence spectra of 2,6-PPYPV-(2+) in methanol solution with different metal ions. 

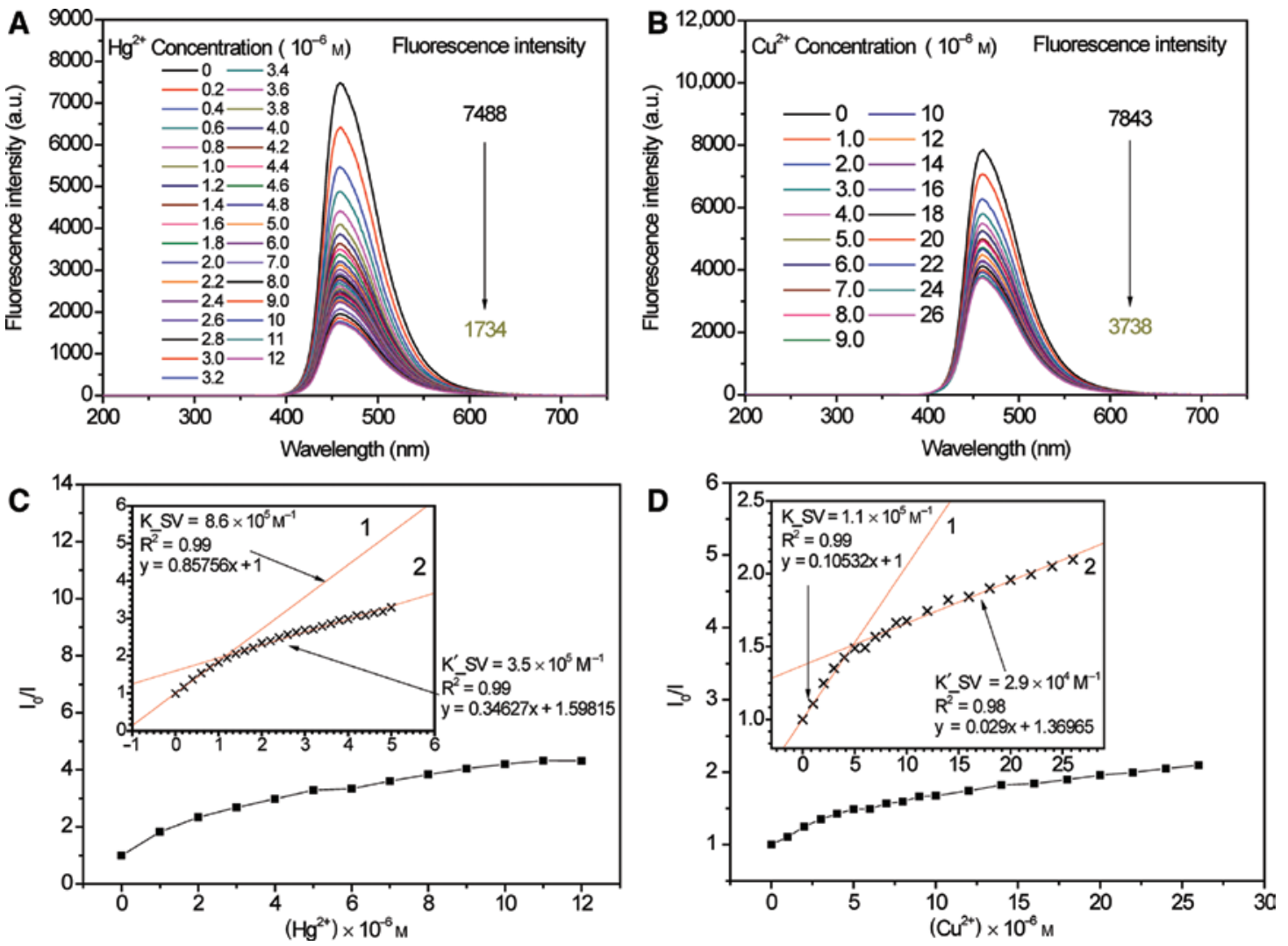

Figure 3: The fluorescence quenching analysis of 2,6-PPYPV-(2+) in aqueous solution, including fluorescence changes at different concentrations and K_SV curves.

(A) Fluorescence spectra of 2,6-PPYPV-(2+) with different concentrations of $\mathrm{Hg}^{2+}$ in aqueous solution. (B) Fluorescence spectra of 2,6-PPYPV(2+) with different concentrations of $\mathrm{Cu}^{2+}$ in aqueous solution. (C) Plot of fluorescence quenching efficiencies $\left(\mathrm{I}_{0} / \mathrm{l}\right)$ versus concentration of $\mathrm{Hg}^{2+}$. (D) Plot of fluorescence quenching efficiencies $\left(\mathrm{I}_{0} / \mathrm{I}\right)$ versus concentration of $\mathrm{Cu}^{2+}$.

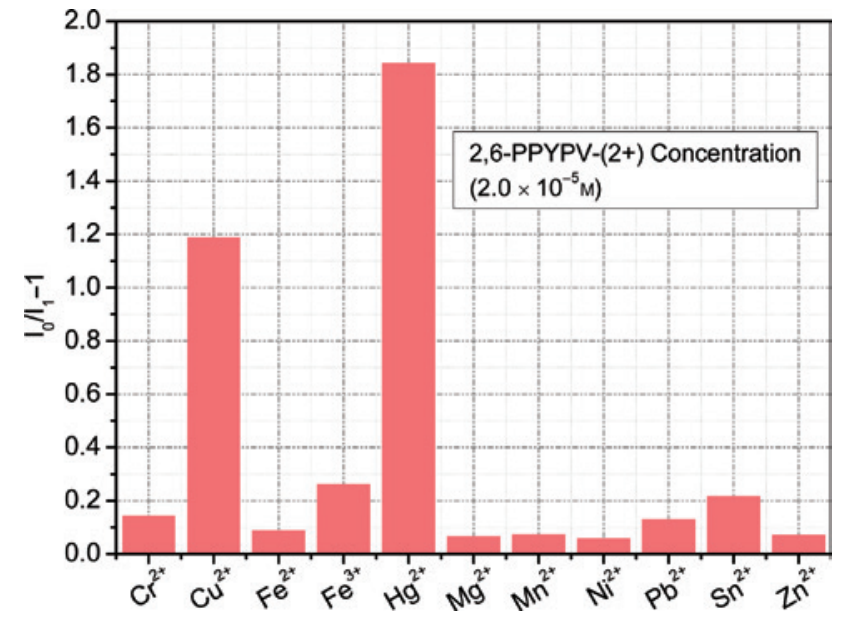

Figure 4: The fluorescence-quenching degrees of 2,6-PPYPV-(2+) with different metal ions in aqueous solution (metal ions concentration $=1.2 \times 10^{-5} \mathrm{M}$ ).

these results, it is certain that the Stern-Volmer constants (K_SV) of the polymer-Cu complex are smaller than that of the polymer-Hg complex, and this result indicates the
CPEs 2,6-PPYPV-(2+) exhibit stronger recognition capability of $\mathrm{Hg}^{2+}$ than that of $\mathrm{Cu}^{2+}$ in aqueous solution.

Meanwhile, the fluorescence-quenching degrees $\left(\mathrm{I}_{0} /\right.$ $\left.I_{1}-1\right)$ of 2,6-PPYPV-(2+) with different metal ions are shown in Figure 4, where $I_{0}$ and $I_{1}$ are the PL intensity of 2,6PPYPV-(2+) in the absence and in the saturation of metal ions, respectively. All the concentrations of these metal ions are $1.2 \times 10^{-5} \mathrm{M}$ which is the fluorescence-quenching extreme limit of $\mathrm{Hg}^{2+}$. It is shown that $\mathrm{Hg}^{2+}$ had a highest fluorescence-quenching degree around 1.84. $\mathrm{Cu}^{2+}$ also has a high quenching degree around 1.19, and other metal ions have ignorable quenching degrees $(0.060-0.26)$. These results show that 2,6-PPYPV-(2+) exhibits great selectivity and sensitivity of $\mathrm{Hg}^{2+}$ and $\mathrm{Cu}^{2+}$ ions in the aqueous phase.

The investigation of 2,6-PPYPV-(2+) in methanol solution is same as in aqueous solution. Figure 5A shows the fluorescence spectra of 2,6-PPYPV-(2+) with different concentrations of $\mathrm{Pd}^{2+}$ in methanol solution. It is indicated that 2,6-PPYPV-(2+) has a wonderful identification ability for $\mathrm{Pd}^{2+}$. When the concentration of $\mathrm{Pd}^{2+}$ was only $8.0 \times 10^{-6} \mathrm{M}$, the fluorescence intensity of 2,6-PPYPV-(2+) 

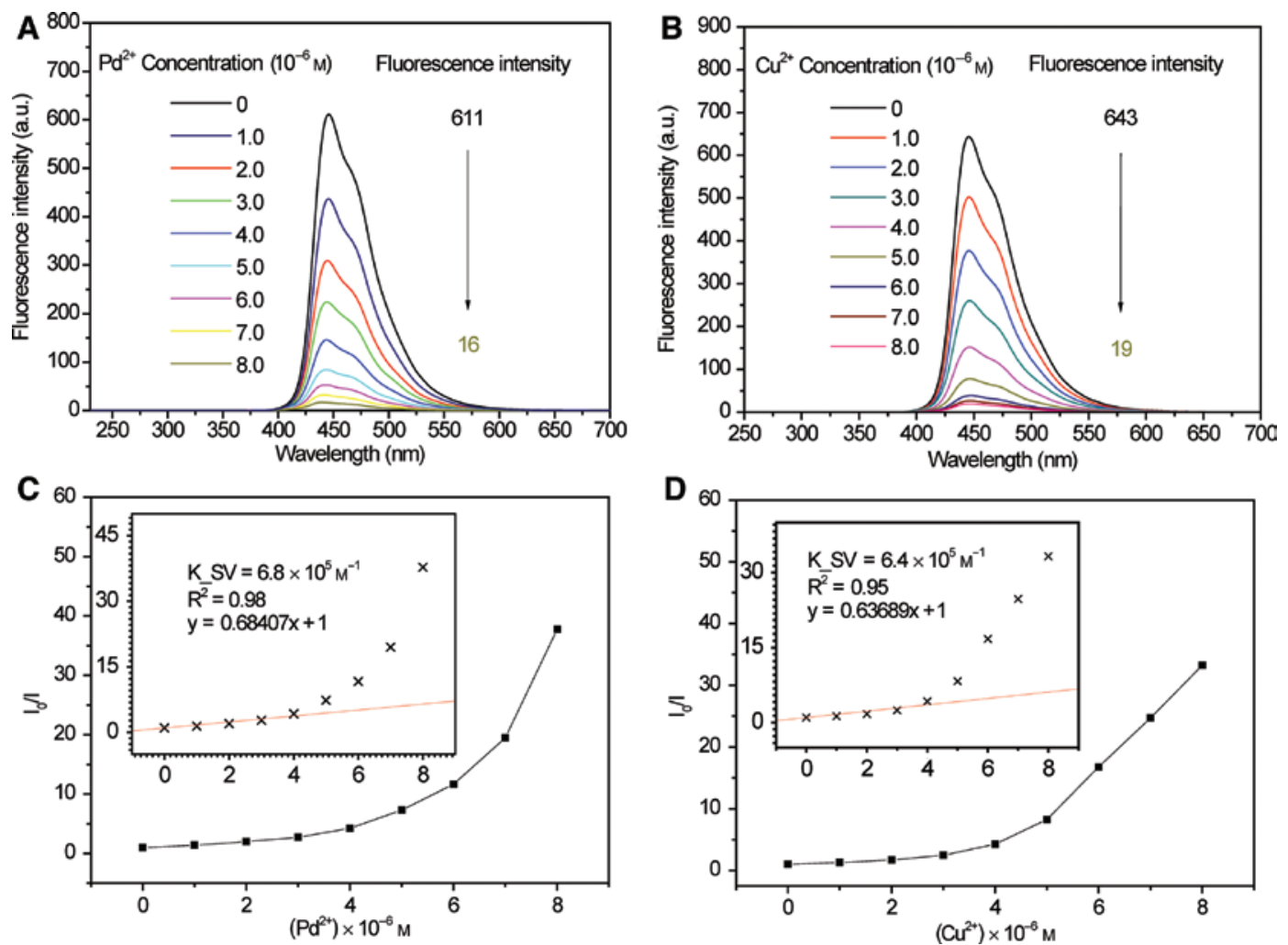

Figure 5: The fluorescence quenching analysis of 2,6-PPYPV-(2+) in methanol solution, including fluorescence changes at different concentrations and K_SV curves.

(A) Fluorescence spectra of 2,6-PPYPV-(2+) with different concentrations of $\mathrm{Pd}^{2+}$ in methanol solution. (B) Fluorescence spectra of 2,6-PPYPV(2+) with different concentrations of $\mathrm{Cu}^{2+}$ in methanol solution. (C) Plot of fluorescence quenching efficiencies $\left(\mathrm{I}_{0} / \mathrm{I}\right)$ versus concentration of $\mathrm{Pd}^{2+}$. (D) Plot of fluorescence quenching efficiencies $\left(\mathrm{I}_{0} / \mathrm{I}\right)$ versus concentration of $\mathrm{Cu}^{2+}$.

decreased from 611 to 16 and about $97 \%$ of fluorescence is quenched. 2,6-PPYPV-(2+) also has a wonderful identification ability for $\mathrm{Cu}^{2+}$ as same as $\mathrm{Pd}^{2+}$. As shown in Figure $5 \mathrm{~B}$, the saturated concentration of $\mathrm{Cu}^{2+}$ which almost completely quenches the fluorescence of 2,6-PPYPV-(2+) reaches to $8.0 \times 10^{-6} \mathrm{M}$.

In addition, plots of fluorescence quenching efficiencies $\left(\mathrm{I}_{0} / \mathrm{I}\right)$ versus $\mathrm{Pd}^{2+}$ and $\mathrm{Cu}^{2+}$ concentration are shown in Figure $5 \mathrm{C}$ and $\mathrm{D}$, respectively. At low concentration of ions $\left(0-4.0 \times 10^{-6} \mathrm{M}\right)$, the plots are fitted by standard Stern-Volmer equations with the K_SV values about $6.8 \times 10^{5} \mathrm{M}^{-1}$ for $\mathrm{Pd}^{2+}$ and $6.4 \times 10^{5} \mathrm{M}^{-1}$ for $\mathrm{Cu}^{2+}$, respectively. Certainly, the K_SV of $\mathrm{Cu}^{2+}$ in methanol solution is about 6 times larger than the K_SV of $\mathrm{Cu}^{2+}$ in aqueous solution and it means the aggregation of polyelectrolytes molecules in water may influence the binding between metal ions and CPEs.

Thus, 2,6-PPYPV-(2+) have a stronger identification ability in methanol. In contrast, 2,6-PPYPV-(2+) cannot recognize $\mathrm{Hg}^{2+}$ in methanol although it has a wonderful identification ability with the satisfying K_SV value about $8.6 \times 10^{-5} \mathrm{M}^{-1}$ in aqueous solution. On the other hand, generally, the Stern-Volmer plots are found to be linear, in which the quenching mechanism is mainly due to the dynamic or static process. However, in some particular cases, especially for CPEs, the experimental results show

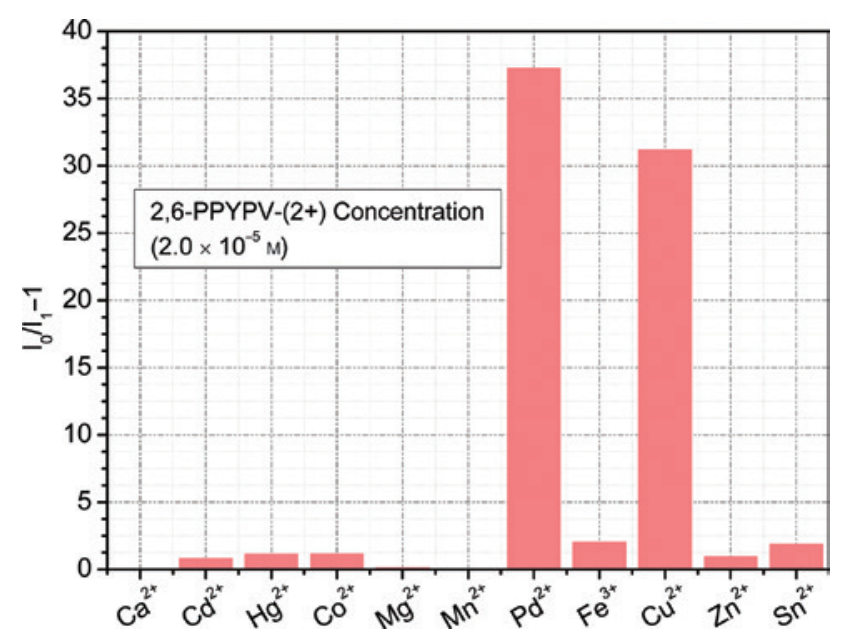

Figure 6: The fluorescence-quenching degrees of 2,6-PPYPV-(2+) with different metal ions in methanol solution (metal ions concentration $=8.0 \times 10^{-6} \mathrm{M}$ ). 

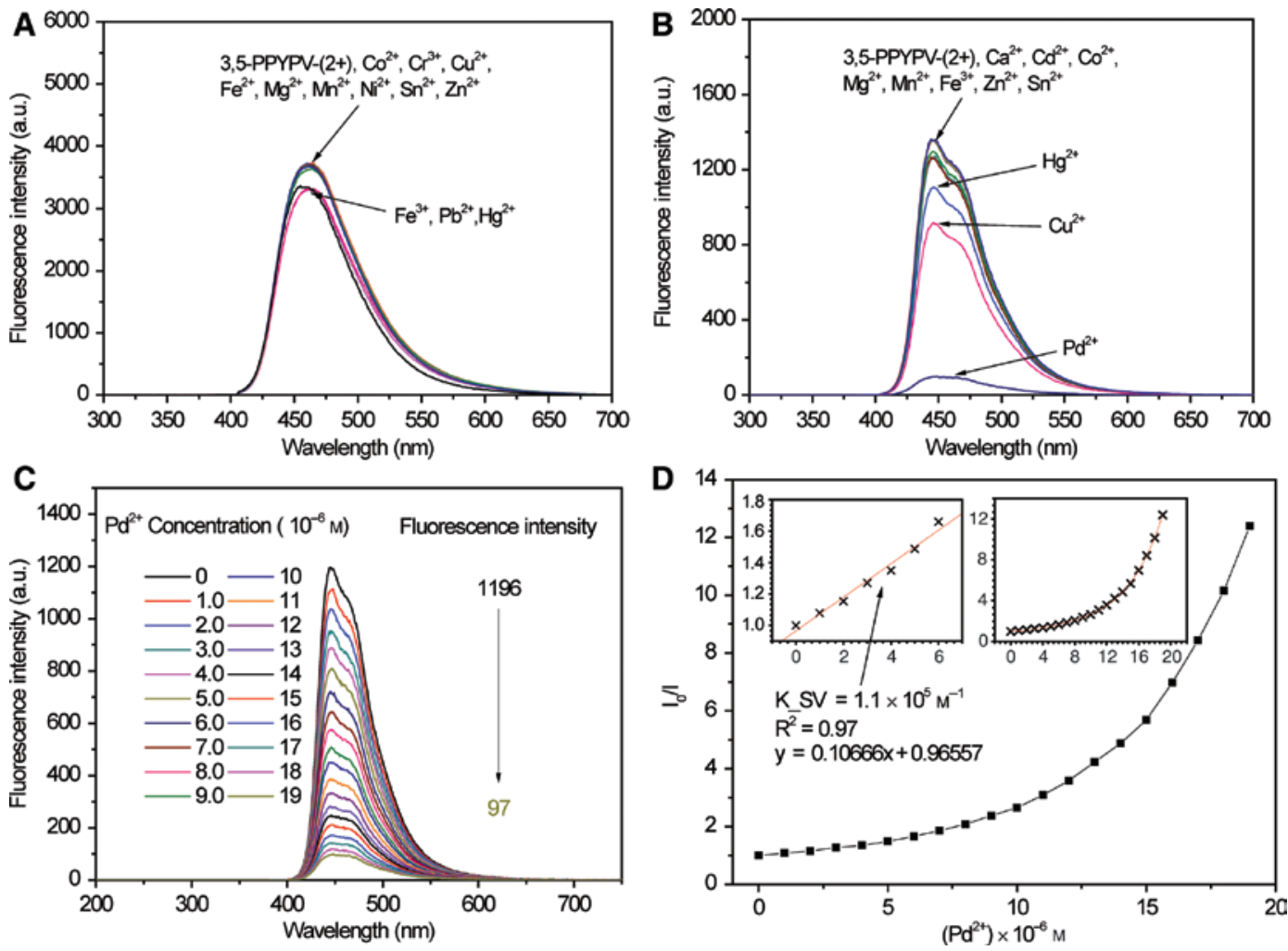

Figure 7: No response for 3,5-PPVPV-(2+) in aqueous solution and $\mathrm{Pd}^{2+}$ can nearly quench the entire fluorescence in methanol solution. (A) Fluorescence spectra of 3,5-PPYPV-(2+) in aqueous solution with different metal ions. (B) Fluorescence spectra of 3,5-PPYPV-(2+) in methanol solution with different metal ions. (C) Fluorescence spectra of 3,5-PPYPV-(2+) with different concentrations of Pd ${ }^{2+}$. (D) Plot of fluorescence quenching efficiencies $\left(\mathrm{I}_{0} / \mathrm{l}\right)$ versus concentration of $\mathrm{Pd}^{2+}$.

positive deviation from the linear Stern-Volmer relation, which is caused by the combination of static quenching and dynamic quenching $(30,31)$, so the Stern-Volmer plot cannot be described with the normal Stern-Volmer equation. As shown in Figure $5 \mathrm{C}$ and D, both plots show positive deviations which further suggest that 2,6-PPYPV-(2+) is a typical conjugated polyelectrolyte. Similarly, the fluorescence-quenching degrees of 2,6-PPYPV-(2+) with different metal ions are given in Figure 6. All the concentrations of these metal ions are $8.0 \times 10^{-6} \mathrm{M}$ which is the fluorescencequenching extreme limit of $\mathrm{Pd}^{2+}$. It is shown that $\mathrm{Pd}^{2+}$ have a highest fluorescence-quenching degree about 37. $\mathrm{Cu}^{2+}$ also has a high quenching degree about 31 , but other metal ions have ignorable quenching degrees (1-2.5). These results show that 2,6-PPYPV-(2+) exhibits great selectivity and sensitivity of $\mathrm{Pd}^{2+}$ and $\mathrm{Cu}^{2+}$ ions in methanol solution.

It is shown that the fluorescence-quenching degrees of $\mathrm{Pd}^{2+}$ and $\mathrm{Cu}^{2+}$ are much higher than those of other metal ions. And the K_SV values for these two metal ions are at the same order of magnitude with PPE type conjugated polymer based on meta-pyridine (21), but CPEs are watersoluble and can realize fluorescence sensing in water system compared to oil-soluble conjugated polymer.
Furthermore, the fluorescence-quenching degrees in methanol solution are generally bigger than that of aqueous solution. All of these results indicated that 2,6PPYPV-(2+) could recognize $\mathrm{Pd}^{2+}$ and $\mathrm{Cu}^{2+}$ in methanol solution.

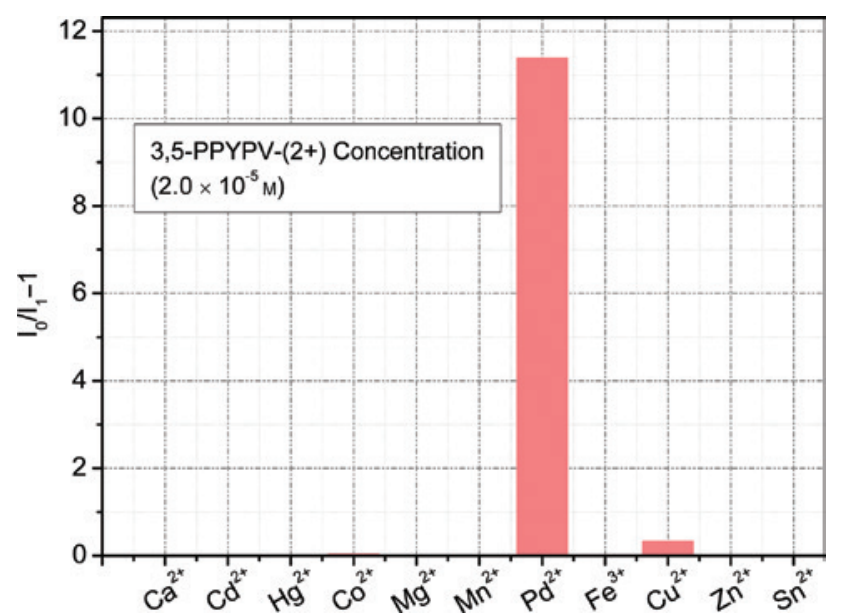

Figure 8: The fluorescence-quenching degrees of 3,5-PPYPV-(2+) with different metal ions in methanol solution (metal ions concentration $=1.9 \times 10^{-5} \mathrm{M}$ ). 

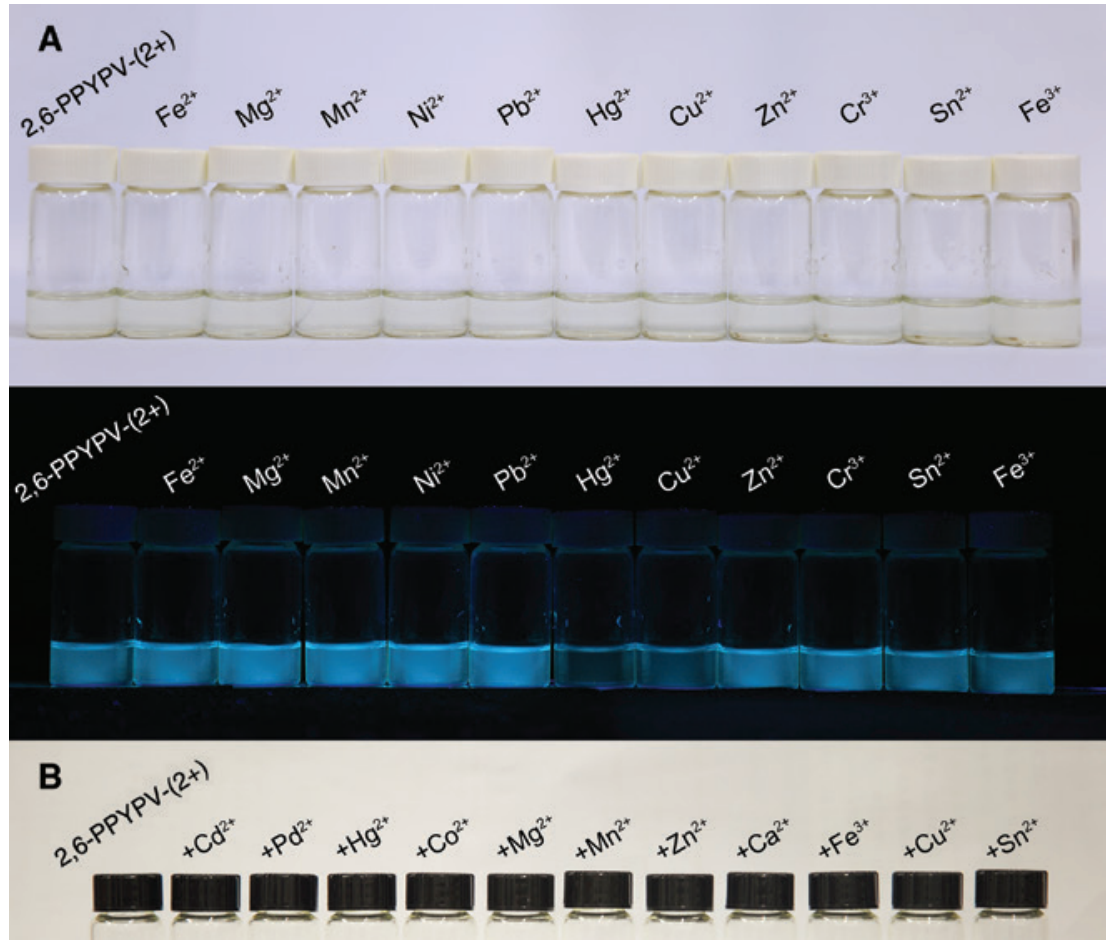

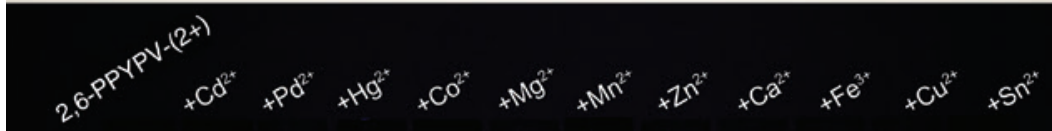
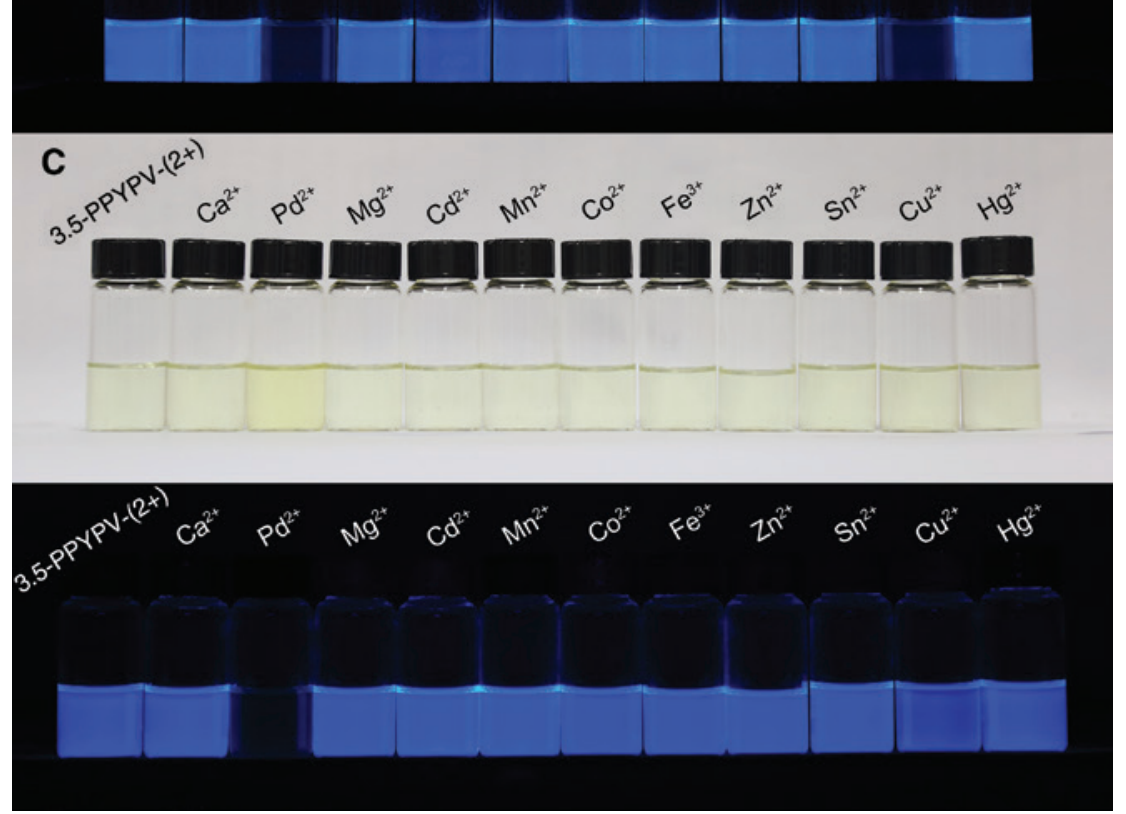

Figure 9: The color changes of the solutions in daylight and fluorescence changes under ultraviolet can be easily observed. The colorimetric photos of (A) aqueous solutions of 2,6-PPYPV-(2+) with metal ions. (B) Methanol solutions of 2,6-PPYPV-(2+) with metal ions. (C) Methanol solutions of 3,5-PPYPV-(2+) with metal ions. 


\subsubsection{Sensing properties of 3,5-PPYPV-(2+)}

All the measurement methods of 3,5-PPYPV-(2+) in sensing properties are the same as 2,6-PPYPV-(2+). Unfortunately, as the result shows in Figure 7A, there are no special responses for common ions in aqueous solution of 3,5-PPYPV-(2+), and only $\mathrm{Fe}^{3+}, \mathrm{Pb}^{2+}$ and $\mathrm{Hg}^{2+}$ cause slight decreases of the fluorescence intensity. However, with the presence of $\mathrm{Pd}^{2+}$, the emission intensity of the polymer decreases dramatically in methanol solution (The concentration of all metal ions is $1.9 \times 10^{-5} \mathrm{M}$.) $\mathrm{Cu}^{2+}$ and $\mathrm{Hg}^{2+}$ result in slight fluorescence quenching as well. And other metal ions $\left(\mathrm{Co}^{2+}, \mathrm{Cu}^{2+}, \mathrm{Fe}^{3+}, \mathrm{Mg}^{2+}, \mathrm{Mn}^{2+}, \mathrm{Cd}^{2+}\right.$, $\mathrm{Hg}^{2+}, \mathrm{Ca}^{2+}, \mathrm{Sn}^{2+}$ and $\mathrm{Zn}^{2+}$ ) cannot lead to the same phenomenon (see Figure 7B).

Furthermore, as shown in Figure 7C, with the addition of $\mathrm{Pd}^{2+}$, the fluorescent intensity is gradually and obviously decreased. When $\mathrm{Pd}^{2+}$ concentration reaches $2.0 \times 10^{-5} \mathrm{M}$, the fluorescence intensity has been changed from 1196 to 97 and about $92 \%$ of fluorescence is quenched.

Similar to situations of 2,6-PPYPV-(2+) with $\mathrm{Pd}^{2+}$ and $\mathrm{Cu}^{2+}$ in methanol solution, the Stern-Volmer plot of 3,5PPYPV-(2+) with $\mathrm{Pd}^{2+}$ in methanol solution shows positive deviation as well, which means the mechanism of quenching with $\mathrm{Pd}^{2+}$ is the combination of static quenching and dynamic quenching. However, at the low concentration $\left(0-6.0 \times 10^{-6} \mathrm{M}\right)$, fluorescence quenching usually introduce static or dynamic quenching instead of the combined effect. Thus, Stern-Volmer plot exhibits a very linear relationship and it can be described as the equation, $\mathrm{y}=0.10666 \mathrm{x}+0.96557$, with a $\mathrm{K} \_\mathrm{SV}$ value of $1.1 \times 10^{5} \mathrm{M}^{-1}$ at low concentration $\left(0-6.0 \times 10^{-6} \mathrm{M}\right)$. The $\mathrm{K} \_S V$ value is larger than $2.97 \times 10^{4} \mathrm{M}^{-1}$, which is the $\mathrm{K} \_S V$ value of CPEs based on benzoselenadiazole (28). This comparison indicates 3,5-PPYPV-(2+) has a favorable selectivity for $\mathrm{Pd}^{2+}$ in methanol solution. Certainly, to effectively describe relationships of $\mathrm{I}_{0} / \mathrm{I}$ versus $\mathrm{Pd}^{2+}$ concentration, we use a quartic equation with one unknown parameter to fit the Stern-Volmer plot and finally we obtain a very satisfactory equation with an $\mathrm{R}^{2}$ value of 0.99 . The definite equation is shown as $y=1.05-0.0323 x+0.0399 x^{2}-0.00389 x^{3}$ $+0.000185 \mathrm{x}^{4}$.

In addition, the fluorescence-quenching degrees of 3,5-PPYPV-(2+) with different metal ions are shown in Figure 8. Obviously, fluorescence-quenching degrees of metal ions are almost 0 except $\mathrm{Pd}^{2+}$ and $\mathrm{Cu}^{2+}$. Even if 3,5PPYPV-(2+) has a non-ignorable fluorescence-quenching degree for $\mathrm{Cu}^{2+}$, the value for $\mathrm{Pd}^{2+}$ is much larger than it.

Furthermore, colorimetric sensing by naked eyes is the simplest way to observe the response signal of sensors for some metal ions. With the purpose to implement quick, convenient and cheap sensing, the colorimetric phenomenon was recorded through photos (see Figure 9). All the polymer concentrations are $2.0 \times 10^{-5} \mathrm{M}$ and metal concentrations are saturated.

In the photo of aqueous solutions of 2,6-PPYPV-(2+) with metal ions, these solutions do not have any difference from each other under daylight, but under exposure to ultraviolet light, the color of solutions in the presence of $\mathrm{Hg}^{2+}$ and $\mathrm{Cu}^{2+}$ changed. However, under the daylight, the methanol solutions of 2,6-PPYPV-(2+) with $\mathrm{Pd}^{2+}$ and $\mathrm{Cu}^{2+}$ changed from colorless to light brown. According to this phenomenon, it could be concluded that static quenching is existent in these mixture which is caused by the formation of polymer-metal complex. Under UV excitation, these two solutions hardly emit any visible fluorescence and other solutions exhibit a strong blue fluorescence. The methanol solutions of 3,5-PPYPV-(2+) exhibits the similar phenomenon as the solutions of 2,6-PPYPV-(2+) and the only difference among these solutions is that the color change of 3,5-PPYPV-(2+) with $\mathrm{Cu}^{2+}$ is less visible than that of 2,6-PPYPV-(2+). These photos suggest that it is easy to detect metal ions such as $\mathrm{Hg}^{2+}$, $\mathrm{Pd}^{2+}$ and $\mathrm{Cu}^{2+}$ with these two novel conjugated polyelectrolyte solutions with the naked eye.

\section{Conclusions}

In summary, two novel meta-linked CPEs 3,5-PPYPV-(2+) and 2,6-PPYPV-(2+) have been designed, synthesized and characterized. From the aspect of metal sensing, $\mathrm{Hg}^{2+}$ and $\mathrm{Cu}^{2+}$ can obviously quench the fluorescence of 2,6-PPYPV-(2+) in aqueous solution and the plots of fluorescence quenching efficiencies $\left(\mathrm{I}_{0} / \mathrm{I}\right)$ versus concentration of $\mathrm{Cu}^{2+}$ and $\mathrm{Hg}^{2+}$ possess a very linear interrelationship which complies with the Stern-Volmer equation at the low concentration of ions. The calculated K_SV of $\mathrm{Cu}^{2+}$ and $\mathrm{Hg}^{2+}$ are $1.1 \times 10^{5} \mathrm{M}^{-1}$ and $8.6 \times 10^{5} \mathrm{M}^{-1}$, respectively. In methanol solution, $\mathrm{Hg}^{2+}$ can no longer quench the fluorescence. Instead, $\mathrm{Pd}^{2+}$ and $\mathrm{Cu}^{2+}$ can drastically reduce the fluorescence intensity of the polymer. The $\mathrm{K} \_\mathrm{SV}$ of $\mathrm{Cu}^{2+}$ reach to $6.4 \times 10^{5} \mathrm{M}^{-1}$ and become larger compared to the value in an aqueous solution. $\mathrm{Pd}^{2+}$ has strong quenching ability as well as $\mathrm{Cu}^{2+}$ with the value of K_SV about $6.8 \times 10^{5} \mathrm{M}^{-1}$. All the results mentioned indicate that 2,6-PPYPV-(2+) has satisfactory recognition capability for $\mathrm{Hg}^{2+}$, $\mathrm{Cu}^{2+}$ in aqueous solution and for $\mathrm{Pd}^{2+}, \mathrm{Cu}^{2+}$ in methanol solution, respectively. As for 3,5PPYPV-(2+), no metal ions can influence the fluorescence emission in aqueous solution. However, in methanol 
solution, $\mathrm{Pd}^{2+}$ can completely quench the fluorescence of 3,5-PPYPV-(2+) and other metal ions do not cause any obvious changes of the emission. Moreover, the plot of fluorescence quenching efficiencies $\left(\mathrm{I}_{0} / \mathrm{I}\right)$ the versus concentration of $\mathrm{Pd}^{2+}$ can be faultlessly fitted with a quartic equation. It is certain that 3,5-PPYPV-(2+) is an excellent material for exclusively detecting $\mathrm{Pd}^{2+}$. Finally, the colorimetric photos of polymer solutions with ions show that the two polymers have considerable potential to become effective analytical tools of fluorescence chemosensor for metal ions.

Acknowledgments: This work is financially supported by the Natural Science Foundation of Sichuan Provincial Education-Department (Grant no. 16ZB0052), the "Shuangzhi" Project of Sichuan Agricultural University (No. 00770105) and the National University Student Innovation Program (No. 201710626019).

\section{References}

1. Jiang H, Taranekar P, Reynolds JR, Schanze KS. Conjugated polyelectrolytes: synthesis, photophysics, and applications. Angew Chem Int Ed. 2009;48(24):4300-16.

2. Yang R, Xu Y, Dang X-D, Nguyen T-Q, Cao Y, Bazan GC. Conjugated oligoelectrolyte electron transport/injection layers for organic optoelectronic devices. J Am Chem Soc. 2008;130(11):3282-3.

3. Taranekar P, Qiao Q, Jiang H, Ghiviriga I, Schanze KS, Reynolds JR. Hyperbranched conjugated polyelectrolyte bilayers for solarcell applications. J Am Chem Soc. 2007;129(29):8958-9.

4. Feng F, Wang H, Han L, Wang S. Fluorescent conjugated polyelectrolyte as an indicator for convenient detection of DNA methylation. J Am Chem Soc. 2008;130(34):11338-43.

5. Jiang $\mathrm{H}$, Zhao X, Schanze KS. Effects of polymer aggregation and quencher size on amplified fluorescence quenching of conjugated polyelectrolytes. Langmuir 2007;23(18):9481-6.

6. Pu KY, Liu B. Conjugated polyelectrolytes as light-up macromolecular probes for heparin sensing. Adv Funct Mater. 2009;19(2):277-84.

7. Pu KY, Liu B. Fluorescent conjugated polyelectrolytes for bioimaging. Adv Funct Mater. 2011;21(18):3408-23.

8. Zhou Q, Swager TM. Fluorescent chemosensors based on energy migration in conjugated polymers: the molecular wire approach to increased sensitivity. J Am Chem Soc. 1995;117(50):12593-602.

9. Ding W, Xu J, Wen Y, Zhang H, Zhang J. Facile fabrication of fluorescent poly (5-cyanoindole) thin film sensor via electropolymerization for detection of Fe $3+$ in aqueous solution. J Photochem Photobiol A Chem. 2016;314:22-8.

10. Xia F, Zuo X, Yang R, Xiao Y, Kang D, Vallée-Bélisle A, Gong X, Yuen JD, Hsu BB, Heeger AJ, Plaxco KW. Colorimetric detection of DNA, small molecules, proteins, and ions using unmodified gold nanoparticles and conjugated polyelectrolytes. Proc Natl Acad Sci USA. 2010;107(24):10837-41.
11. Wang D, Gong X, Heeger PS, Rininsland F, Bazan GC, Heeger AJ. Biosensors from conjugated polyelectrolyte complexes. Proc Natl Acad Sci USA. 2002;99(1):49-53.

12. Zhu X, Xiao Y, Jiang X, Li J, Qin H, Huang H, Zhang Y, He X, Wang $\mathrm{K}$. A ratiometric nanosensor based on conjugated polyelectrolyte-stabilized AgNPs for ultrasensitive fluorescent and colorimetric sensing of melamine. Talanta 2016;151:68-74.

13. DiCesare N, Pinto MR, Schanze KS, Lakowicz JR. Saccharide detection based on the amplified fluorescence quenching of a water-soluble poly (phenylene ethynylene) by a boronic acid functionalized benzyl viologen derivative. Langmuir 2002;18(21):7785-7.

14. Fan L-J, Zhang Y, Murphy CB, Angell SE, Parker MF, Flynn BR, Jones WE. Fluorescent conjugated polymer molecular wire chemosensors for transition metal ion recognition and signaling. Coord Chem Rev. 2009;253(3):410-22.

15. Huo L, Zhang S, Guo X, Xu F, Li Y, Hou J. Replacing alkoxy groups with alkylthienyl groups: a feasible approach to improve the properties of photovoltaic polymers. Angew Chem. 2011;123(41):9871-6.

16. Li Y, Huang H, Li Y, Su X. Highly sensitive fluorescent sensor for mercury (II) ion based on layer-by-layer self-assembled films fabricated with water-soluble fluorescent conjugated polymer. Sens Actuators B Chem. 2013;188:772-7.

17. Tan C, Pinto MR, Schanze KS. Photophysics, aggregation and amplified quenching of a water-soluble poly (phenylene ethynylene). Chem Commun. 2002;(5):446-7.

18. Li J, Meng J, Huang X, Cheng Y, Zhu C. A highly selective fluorescent sensor for $\mathrm{Hg} 2+$ based on the water-soluble poly (p-phenyleneethynylene). Polymer 2010;51(15):3425-30.

19. Gao Y, Wang C-C, Wang L, Wang H-L. Conjugated polyelectrolytes with $\mathrm{pH}$-dependent conformations and optical properties. Langmuir 2007;23(14):7760-7.

20. Kou C, He X, Jiang X, Ni Y, Liu L, Huangfu C, Liu K. Novel isoindigo-based conjugated polyelectrolytes: synthesis and fluorescence quenching behavior with water-soluble poly (p-phenylenevinylene) s. J Polym Sci Part A: Polym Chem. 2015;53(19):2223-37.

21. Huang H, Wang K, Tan W, An D, Yang X, Huang S, Zhai Q, Zhou L, Jin Y. Design of a modular-based fluorescent conjugated polymer for selective sensing. Angew Chem Int Ed. 2004;43(42):5635-8.

22. Pinto MR, Schanze KS. Conjugated polyelectrolytes: synthesis and applications. Synthesis 2002;2002(09):1293-309.

23. Liu B, Yu W-L, Lai Y-H, Huang W. Blue-light-emitting cationic water-soluble polyfluorene derivatives with tunable quaternization degree. Macromolecules 2002;35(13):4975-82.

24. Fan Q-L, Zhou Y, Lu X-M, Hou X-Y, Huang W. Water-soluble cationic poly ( $p$-phenyleneethynylene)s (PPEs): effects of acidity and ionic strength on optical behavior. Macromolecules 2005;38(7):2927-36.

25. Kaur P, Yue H, Wu M, Liu M, Treece J, Waldeck DH, Xue C, Liu H. Solvation and aggregation of polyphenylethynylene based anionic polyelectrolytes in dilute solutions. J Phys Chem B. 2007;111(29):8589-96.

26. Dou W, Wang C, Wang G, Ma Q, Su X. Enhance effect of surfactants on the photoluminescence and photostability of water-soluble poly (phenylene ethynylene). J Phys Chem B. 2008;112(40):12681-5.

27. Xiang G, Cui W, Lin S, Wang L, Meier H, Li L, Cao D. A conjugated polymer with ethyl 2-(2-(pyridin-2-yl)-1H-benzo[d]imidazol-1-yl) 
acetate units as a novel fluorescent chemosensor for silver (I) detection. Sens Actuators B Chem. 2013;186:741-9.

28. Lei Y, Li H, Huang X, Chen J, Liu M, Gao W, Ding J, Lin D, Wu H. Water-soluble benzoselenadiazole-based conjugated polymer fluorescent sensor with high selectivity for ferric ions and mercury ions and possible applications as integrated molecular logic gates. Tetrahedron 2015;71(21):3453-62.

29. Makarska-Bialokoz M. Interactions of hemin with bovine serum albumin and human hemoglobin: a fluorescence quenching study. Spectrochim Acta A Mol Biomol Spectrosc. 2018;193: 23-32.

30. Haskins-Glusac K, Pinto MR, Tan C, Schanze KS. Luminescence quenching of a phosphorescent conjugated polyelectrolyte. J Am Chem Soc. 2004;126(45):14964-71.

31. López Cabarcos E, Carter SA. Characterization of the photoluminescence quenching of mixed water-soluble conjugated polymers for potential use as biosensor materials. Macromolecules 2005;38(10):4409-15. 